\title{
Kondo resonance in a multi-probe quantum dot
}

\author{
Qing-feng Sun and Hong Guo \\ Center for the Physics of Materials and Department of Physics, McGill University, Montreal, PQ, Canada H3A $2 T 8$
}

We present a theoretical analysis of a possible route for directly detecting Kondo resonances in local density of states (LDOS) of an interacting quantum dot. By very weakly couple a third and/or a fourth lead to a two-probe quantum dot and measuring differential conductance through these extra links, we show that Kondo peaks directly map onto the differential conductance measured from the third link. We analyze the conditions by which this detection of Kondo peaks in LDOS is possible.

73.40.Gk, 72.15.Qm, 73.20.At

The Kondo effect is a prototypical many-body correlation effect in condensed matter involving the interaction between a localized spin and free electrons. At low temperatures and in the Kondo regime, the entire systemlocalized spin plus free electrons, forms a spin singlet state and the localized spin is screened. As a result, a very narrow Kondo resonance peak arises in the local density of states (LDOS) at the chemical potential of the free electrons. The Kondo effect was first discovered in metals containing a small amount of magnetic impurities. More receptly it was observed in semiconductor quantum dots (QD)1 13 which has generated a great deal of theoretical and experimental interests because it provides rich understanding to many-body phenomena at the mesoscopic scale.

Experimental investigations of Kondo phenomenon in semiconfuctor QD were mainly through two observations 4 . First, for cases of QD confining an odd number of electrons, the differential conductance $d I / d V$ is measured as a function of a gate voltage $V_{g}$, here $I$ is the two-probe tunneling current and $V$ the bias voltage. It was found that $d I / d V$ in the Coulomb blockade region is enhanced 12 due to the Kondo effect. Second, there is a peak at bias $V=0$ in the $d I / d V$ versus $V$ curve, and this peak splits into two when there is a magnetic field 1 . Although $d I / d V$ gives a measure of LDOS of the QD in linear response, to the best of our knowledge the comprehensive shape of the LDOS of the QD in the Kondo regime, namely the one (or a few) narrow Kondo peak on top of the "shoulder" of the broad peak corresponding to a intradot level, has so far not been directly detected in any experiment.

The outstanding features in the QD Kondo phenomenon are most prominent and plentiful in LDOS than in the tunneling current $I$ and its associated differential conductance $d I / d V$. For example, for a QD coupled to a normal lead and a superconducting lead (a N-QD-S device), three Kondo peaks arise in LDOS at the chem- ical potential of the normal lead and at the superconducting gap $( \pm \Delta)$ respectively. On the other hand, the tunneling current hardly vary atall despite the presence of these Kondo peaks in LDOSB. As another example, in an asymmetric N-QD-S device under a finite on-site Coulomb interaction and a large superconducting gap, four Kondo peaks emerge in LDOst. However, while the current is enhanced due to these features, it does not show clear characteristics of the narrow Kondo resonances. Therefore, it is extremely useful to be able to directly detect the narrow Kondo resonance in the LDOS.

Given the importance of the physics of Kondo phenomenon in mesoscopic systems and the extensive investigations in both theory and experiments, it is indeed surprising to see the lack of direct observation of the Kondo resonance peaks in the QD LDOS5. It is the purpose of this communication to present a theoretical analysis of a possible route for solving this problem. Because, as discussed above, that two-probe tunneling differential conductance $d I / d V$ does not reveal the narrow Kondo peaks in LDOS, we will investigate a new approach by which one or two extra leads are used to probe the QD. When conditions are controlled correctly, we show that the LDOS (including the narrow Kondo peaks) will directly map onto the current measured at the extra leads thereby providing a direct measurements of the narrow Kondo peaks elusive so far.

To begin, let's consider the hypothetical device consisting of a QD coupled to four leads fabricated by a split gate technique, as shown in the inset of Fig.2, in a two-dimensional electron gas (2DEG). Here, leads 1 and 3 plus the QD form a typical two-probe QD device for which we assume as having a Kondo regime at low temperature, so that there are some Kondo resonances in the LDOS which is our target of measurement. Leads 2 and 4 are assumed to very weakly couple with the QD, much weaker than that of leads 1 and 3 . The quantification of these statements will be made below. Our hope is to probe the QD Kondo physics through lead 2. The purpose of the bias on lead $4, V_{4}$, is to provide a voltage opposite in sign to that of $V_{2}$, so as to compensate the intradot energy altered by bias $V_{2}$. Our results suggest that when conditions are right, the differential conductance $d I_{2} / d V_{2}$ versus its terminal bias $V_{2}$, gives an excellent measurement to the LDOS as a function of energy $\epsilon$, thereby allowing us to observe the Kondo peaks directly.

There are so far some literature aftempting to measure the Kondo resonance in LDOS5 7 . They use a scanning tunneling microscope (STM) to obtain spectroscopic data on individual magnetic impurities deposited onto the host metal. However, due to quantum interference 
between the $d$ and/or $f$ orbitals of the magnetic impurity and the continuum conduction electron channels, so far only Fano like resonances were obtained in the spectrum rather than the expected Lorentzian shape of the Kondo peaks. In contrast, our present hypothetical experiment will attempt to directly obtain the original shape of the Kondo resonance in LDOS. The essential difference in our device as compared to those studied before are the following. In the STM experiment 7 , there exists electron transitions between the host metal, which provides conduction or free electrons, and the probing STM tip, such transitions cannot be avoided. In our device, there does not exist direct tunneling between the probe terminal lead 2 and the conduction electron channels lead 1 and 3. This important difference allows us to observe the original shape of LDOS including the Kondo resonances.

Our device is described by the following Hamiltonian,

$$
\begin{gathered}
H=\sum_{\alpha, k, \sigma} \epsilon_{\alpha k} a_{\alpha k \sigma}^{\dagger} a_{\alpha k \sigma}+\sum_{\sigma} \epsilon_{d \sigma} d_{\sigma}^{\dagger} d_{\sigma}+U d_{\uparrow}^{\dagger} d_{\uparrow} d_{\downarrow}^{\dagger} d_{\downarrow} \\
+\sum_{\alpha, k, \sigma}\left(v_{\alpha k} a_{\alpha k \sigma}^{\dagger} d_{\sigma}+\text { H.c. }\right)
\end{gathered}
$$

where $a_{\alpha k \sigma}^{\dagger}\left(a_{\alpha k \sigma}\right)(\alpha=1,2,3,4)$ and $d_{\alpha}^{\dagger}\left(d_{\alpha}\right)$ are creation (annihilation) operators in the lead $\alpha$ and the QD, respectively. The QD includes a single energy level, but having spin index $\sigma$ and intradot Coulomb interaction $U$. To account for a possible magnetic field, we allow $\epsilon_{d \uparrow} \neq \epsilon_{d \downarrow}$. The last term describes the tunneling part of the Hamiltonian, with $v_{\alpha k}$ being the coupling matrix element.

The curregt from lead $\alpha$ flowing into the QD can be expressed as 9 ,

$$
I_{\alpha}=-2 e I m \sum_{\sigma} \int \frac{d \epsilon}{2 \pi} \Gamma_{\alpha}\left\{f_{\alpha}(\epsilon) G_{\sigma}^{r}(\epsilon)+\frac{1}{2} G_{\sigma}^{<}(\epsilon)\right\},
$$

where $\Gamma_{\alpha}(\epsilon)=2 \pi \sum_{k}\left|v_{\alpha k}\right|^{2} \delta\left(\epsilon-\epsilon_{\alpha k}\right) ; f_{\alpha}(\epsilon)$ is the Fermi distribution of lead $\alpha ; G_{\sigma}^{r}(\epsilon)$ and $G_{\sigma}^{<}(\epsilon)$ are the retarded and the Keldysh Green's functions of the QD, they are the Fourier transformation of $G_{\sigma}^{r,<}(t)$, and $G_{\sigma}^{r}(t) \equiv$ $-i \theta(t)<\left\{d_{\sigma}(t), d_{\sigma}^{\dagger}(0)\right\}>, G_{\sigma}^{<}(t) \equiv i<d_{\sigma}^{\dagger}(0) d_{\sigma}(t)>$.

Using the standard equation of motion technique and taking the familiar decoupling approximation 10 , we have solved $G_{\sigma}^{r}(\epsilon)$ to be

$$
G_{\sigma}^{r}(\epsilon)=\frac{1+U A_{\sigma} n_{\bar{\sigma}}}{\epsilon-\epsilon_{d \sigma}-\Sigma_{\sigma}^{(0)}+U A_{\sigma}\left(\Sigma_{\bar{\sigma}}^{(a)}+\Sigma_{\bar{\sigma}}^{(b)}\right)},
$$

where $A_{\sigma}(\epsilon)=\left[\epsilon-\epsilon_{d \sigma}-U-\Sigma_{\sigma}^{(0)}-\Sigma_{\bar{\sigma}}^{(1)}-\Sigma_{\bar{\sigma}}^{(2)}\right]^{-1}$ and $\Sigma_{\sigma}^{(0)}=\sum_{k \alpha}\left|v_{\alpha k}\right|^{2} /\left(\epsilon-\epsilon_{\alpha k}+i 0^{+}\right)$is the lowestorder self-energy which is exactly the retarded self-energy for a noninteraction system; $\Sigma_{\sigma}^{(a)}, \Sigma_{\sigma}^{(b)}, \Sigma_{\sigma}^{(1)}$, and $\Sigma_{\sigma}^{(2)}$ are the higher-order self-energies due to the intradot Coulomb interaction and the tunneling coupling. These higher-order self-energies are derived to be: $\Sigma_{\sigma}^{(a)}=$ $\sum_{k \alpha}\left|v_{\alpha k}\right|^{2} f_{\alpha}\left(\epsilon_{\alpha k}\right) / \epsilon_{\sigma}^{+} ; \quad \Sigma_{\sigma}^{(b)}=\sum_{k \alpha}\left|v_{\alpha k}\right|^{2} f_{\alpha}\left(\epsilon_{\alpha k}\right) / \epsilon_{\sigma}^{-} ;$ $\Sigma_{\sigma}^{(1)}=\sum_{k \alpha}\left|v_{\alpha k}\right|^{2} / \epsilon_{\sigma}^{+} ; \quad \Sigma_{\sigma}^{(2)}=\sum_{k \alpha}\left|v_{\alpha k}\right|^{2} / \epsilon_{\sigma}^{-} ;$here $\epsilon_{\sigma}^{+}=\epsilon+\epsilon_{\alpha k}-\epsilon_{d \sigma}-\epsilon_{d \bar{\sigma}}-U+i 0^{+}$and $\epsilon_{\sigma}^{-}=\epsilon-\epsilon_{\alpha k}-$ $\epsilon_{d \bar{\sigma}}+\epsilon_{d \sigma}+i 0^{+}$. The quantity $n_{\bar{\sigma}}$ in Eq.(3) is the intradot electron occupation number $q$ state $\bar{\sigma}$, which needs to be calculated self-consistently 11 . In the limit of having only two leads, the above results reduces to that of Refs. 10,12 .

The Keldysh Green function $G_{\sigma}^{<}$, for interacting systems, can not be obtained from the equation of motion without introducing additipnal assumptions. We use the standard ansatz due to Ng13,

$$
\Sigma_{\sigma}^{<}(\epsilon)=-\sum_{\alpha} \frac{\Gamma_{\alpha} f_{\alpha}(\epsilon)}{\Gamma}\left(\Sigma_{\sigma}^{r}-\Sigma_{\sigma}^{a}\right),
$$

where $\Gamma=\sum_{\alpha} \Gamma_{\alpha} . \quad \Sigma_{\sigma}^{<}$and $\Sigma_{\sigma}^{r}\left(\Sigma_{\sigma}^{a}\right)$ are the lesser and retarded (advanced) self-energies of the interacting system. Then from the Keldysh equation $G_{\sigma}^{<}=G_{\sigma}^{r} \Sigma_{\sigma}^{<} G_{\sigma}^{a}$ and $G_{\sigma}^{a}=\left(G_{\sigma}^{r}\right)^{*}, G_{\sigma}^{<}$can be obtained straightforwardly. With $G_{\sigma}^{r}$ and $G_{\sigma}^{<}$solved, from Eq.(2) the current can be obtained immediately:

$$
I_{\alpha}=-2 e \sum_{\sigma, \alpha^{\prime}} \int \frac{d \epsilon}{2 \pi} \frac{\Gamma_{\alpha} \Gamma_{\alpha^{\prime}}}{\Gamma}\left[f_{\alpha}(\epsilon)-f_{\alpha^{\prime}}(\epsilon)\right] I m G_{\sigma}^{r} .
$$

In the numerical calculation, we make a few further simplifications: (i) we assume square bands of width $2 W$, so that $\Gamma_{\alpha}(\epsilon)=\Gamma_{\alpha} \theta(W-|\epsilon|)$, with $W=1000 \gg$ $\max \left(k_{B} T, V_{\alpha}, \Gamma_{\alpha}\right)$; (ii) we take the large $U$ limit $U \rightarrow \infty$; (iii) considering that the intradot level $\epsilon_{d \sigma}$ is affected by leads' bias voltage $V_{\alpha}$, we assume this effect to be $\epsilon_{d \sigma}=\epsilon_{d \sigma}(0)+\gamma_{2} V_{2}+\gamma_{4} V_{4}$, with $\gamma_{\alpha}=C_{\alpha} / C$. Here $C_{\alpha}$ is the capacitance between lead $\alpha$ and the $\mathrm{QD}$, and $C$ is the total capacitance of the $\mathrm{QD} ; \epsilon_{d \sigma}(0)$ is the location of the intradot energy level at $V_{2}=V_{4}=0$. We set $V_{4}=-V_{2}$ to offset the level change, so that

$$
\epsilon_{d \sigma}=\epsilon_{d \sigma}(0)+\gamma V_{2}
$$

where $\gamma=\left(C_{2}-C_{4}\right) / C$.

Our objective system is the QD plus leads 1 and 3 , it is recovered by setting $\Gamma_{2}=\Gamma_{4}=0$ so that leads 2 and 4 are decoupled from the QD. The dotted curves of Fig.1 shows the intradot LDOS of the objective system 14 . A broad peak at $\epsilon=-2$ is due to the intradot renormalized level. In nonequilibrium and at zero magnetic field $\left(\epsilon_{d \uparrow}=\epsilon_{d \downarrow}\right)$, there exhibits two narrow Kondo resonance peaks at $\mu_{1}$ and $\mu_{3}$ in the LDOS. With a non-zero magnetic field $\left(\epsilon_{d \uparrow} \neq \epsilon_{d \downarrow}\right)$ and in nonequilibrium, four narrow Kondo peaks emerge at $\mu_{1 / 3} \pm \Delta \epsilon$ where $\Delta \epsilon=\epsilon_{d \downarrow}-\epsilon_{d \uparrow}$ is the level difference. These familiar characters of LDOS have been known 12 in theory, our task to "experimentally" measure them.

Let's now turn on a non-zero $\Gamma_{2}$ and $\Gamma_{4}$. Note that these couplings must be greatly weaker than those of leads 1 and 3 , i.e. $\Gamma_{2}, \Gamma_{4} \ll \Gamma_{1}, \Gamma_{3}$, so that they do not affect the QD significantly. We first consider the $\gamma=0$ 
case for which there is a complete compensation of $V_{2}$ by $V_{4}$ so that the level $\epsilon_{d \sigma}$ does not change with $V_{2}$ by Eq.(6). The differential conductance $d I_{2} / d V_{2}$ versus $V_{2}$ is shown in Fig. 1 by the solid curves, and they overlap almost identically with the dotted curves of the two-probe LDOS (the objective system), so that the dotted curves can not be seen in the fig.1. In other words, the $d I_{2} / d V_{2^{-}}$ $V_{2}$ data and the $L D O S-\epsilon$ data of the objective system map into each other essentially perfectly. To see better the comparison, data in the vicinity of Kondo peaks for zero magnetic field are shown in the inset of Fig.1. Although the Kondo peaks of $d I_{2} / d V_{2}$ is slightly lower than those of LDOS, they not only agree in their position but also in the Lorentzian shape which is a very important characteristic of Kondo phenomenon.

Why does $d I_{2} / d V_{2}$ versus $V_{2}$ give such an excellent mapping of the original LDOS of the objective system ? First, lead 2 is very weakly coupled to the QD so that the original QD LDOS is not significantly affected by it. Second, because the QD is coupled to leads 1 and 3 in much stronger way, e.g. $\Gamma_{1}, \Gamma_{3} \gg \Gamma_{2}$, resonance tunneling from lead 2 to leads 1,3 can not occur with any substantial probability. Therefore, an incident electron with energy $\epsilon$ from lead 2 has a probability of tunneling into the QD that is given by the intradot $\operatorname{LDOS}(\epsilon)$, leading to the excellent agreement between $d I_{2} / d V_{2}$ and the LDOS. We conclude that the LDOS versus energy $\epsilon$ can be obtained by measuring $d I_{2} / d V_{2}$ versus $V_{2}$.

It is worth mentioning that although we have assumed symmetric couplings between leads 1,3 to the QD, $\Gamma_{1}=\Gamma_{3}$, and assumed a large e-e interaction $U \rightarrow \infty$, it is straightforward to confirm, as we did, that if $\Gamma_{1} \neq \Gamma_{3}$ and $U$ is finite, our results are still tenable. In fact, these parameters of the objective system only affect its LDOS, they do not destroy the excellent agreement between the signal $d I_{2} / d V_{2}$ and the LDOS. In addition, the physics dictating this excellent agreement is independent of what theoretical methods one uses to derive the Green's functions $G_{\sigma}^{r}(\epsilon)$. In other words, if one uses another method to solve $G_{\sigma}^{r}(\epsilon)$ father than the equation of motion method we used here 12 , or even if one gives an arbitrary LDOS of the target system, our proposed detection technique can still give the excellent agreement between $d I_{2} / d V_{2}$ and the LDOS.

Other important issues concerning our proposal are the ranges of parameters associated with leads 2 and 4 which we use to probe the LDOS. If the resistance provided by lead 1 to the QD is a typical $10 K \Omega$, then if lead 3 couples 1000 times weaker, its contact to QD will have a resistance of $10 M \Omega$ which is experimentally not difficult. When voltages on leads 2 and 4 do not exactly compensate, i.e. when $\gamma \neq 0$, the intradot level $\epsilon_{d \sigma}$ will change with $V_{2}$ according to Eq.(河), hence features in LDOS will be changed which affect the proposed measurement of the Kondo peaks by $d I_{2} / d V_{2}$. Our investigation on this problem shows that this is actually a weak effect on the Kondo resonances, as shown in Fig. 2 where $d I_{2} / d V_{2}$ is plotted against $V_{2}$ at several values of $\gamma$. The background dif- ferential conductance does change with $\gamma$. However, the important result is that the narrow Kondo peaks still keep the original shape, and their locations do not vary at all as shown in Fig.2. Even when $\gamma=0.5$ or larger, these Kondo characters remain. Therefore, we believe the condition on parameter $\gamma$ is not strict for our proposal to work. It should be stressed that if $\gamma$ is not very large, e.g. $\gamma=0.05$, the broad peak which corresponds to the intradot renormalized level, only shifts slightly but retaining its line shape (dashed line of Fig.1). So the comprehensive shape of LDOS in the Kondo regime, the one (or a few) narrow Kondo peak on top of the shoulder of the broad peak, can be detected.

Let's estimate the value of $\gamma$ which, of course, is less than unity. The total capacitance $C$ includes each terminal capacitance $C_{\alpha}(\alpha=1,2,3,4)$, it also includes, perhaps, some gate capacitances $C_{g}$. A gate is a negatively biased metal deposited above the 2DEG while the leads' terminal is in the 2DEG and coupled to QD by tunnel barriers. In general, $C_{\alpha} / C_{\alpha^{\prime}}$ is approximatively proportional to $\Gamma_{\alpha} / \Gamma_{\alpha^{\prime}}$, and $C_{g}$ is larger than $C_{\alpha}$ 15. Because $\Gamma_{2} \ll \Gamma_{1}$ and $\Gamma_{3}$ (in Fig.1 and $2, \Gamma_{1}=\Gamma_{3}=1000 \Gamma_{2}$ ), a conservative estimate is that $C_{1}$ and $C_{3}$ should be larger than $10 C_{2}$. Then, even if we neglect $C_{g}$ in the total $C$ and even if we completely remove the compensating terminal 4 , we still have $\gamma=C_{2} / C<0.05$. Moreover, if the compensating lead 4 is there to cancel the effect of the probe terminal 2 to some extent, we estimate $|\gamma|$ should be less than 0.01 . Hence, by results of Fig.2, such a small $\gamma$ will not cause any trouble to our proposed measurements of the Kondo peaks.

Next, we discuss the range of temperature $T$ where the excellent agreement of $d I_{2} / d V_{2}$ and LDOS can be kept. We fix $\gamma=0.05$, as discussed above, this value of $\gamma$ is easily realized even without the compensating lead 4. Therefore we set, in our theory, $\Gamma_{4}=0$ and $C_{4}=0$, and we propose to replace lead 4 by a gate to control the intradot level position (see inset of Fig.3). The signal $d I_{2} / d V_{2}$ versus $V_{2}$ is shown in Fig.3 for three temperatures. At low temperature, there are four Kondo peaks at $\mu_{1 / 3} \pm \Delta \epsilon$ in $d I_{2} / d V_{2}$, they are the mapping of the four Kondo peaks in $\operatorname{LDOS}(\epsilon)$ which have been discussed above. Increasing temperature causes the Kondo peaks to go down, until they completely disappear. On the other hand, we found that the broad peak which corresponds to the intradot level essentially does not change in this range of temperatures (not shown). These characters are in excellent agreement with those of the LDOS. In fact, our investigations show that when $k_{B} T<\Gamma_{1}+\Gamma_{3}$, the excellent agreements between $d I_{2} / d V_{2}$ and LDOS are always maintained.

To summarize, we have proposed and analyzed a possibility to experimentally directly observe the local density of states of a quantum dot, thereby directly detect Kondo resonance peaks in it. In particular, using an extra weak link to the quantum dot, we showed that curves of $d I_{2} / d V_{2}$ versus $V_{2}$ can map out perfectly the curves of LDOS versus energy, if experimental conditions are set in 
the correct range. We also provided an analysis to these conditions and found them to be reasonable and therefore should be realizable. Indeed, it will be extremely interesting to experimentally test these predictions.

Acknowledgments: We gratefully acknowledge financial support from NSERC of Canada and FCAR of Quebec.

${ }^{1}$ S. M. Cronenwett, T. H. Oosterkamp and L. P. Kouwenhoven, Science 281, 540 (1998); D. Goldhaber-Gordon, H. Shtrikman, D. Mahalu, D.A.-Magder, U. Meirav, and M.A.Kastner, Nature 391, 156 (1998); T. Inoshita, Science 281, 526 (1998).

${ }^{2}$ S. Sasaki, S. De Franceschi, J. M. Elzerman, W. G. van der Wiel, M. Eto, S. Tarucha, and L. P. Kouwenhoven, Nature 405, 764 (2000); W. G. van der Wiel, S. De Franceschi, T. Fujisawa, J. M. Elzerman, S. Tarucha, and L. P. Kouwenhoven, Science 289, 2105 (2000).

${ }^{3}$ A. A. Clerk, V. Ambegaokar and S. Hershfield, Phys. Rev. B 61, 3555 (2000).

${ }^{4}$ Q.-f. Sun, H. Guo and T.-h. Lin, cond-mat/0105120.

${ }^{5}$ V. Madhavan, W. Chen, T. Jamneala, M. F. Crommie, and N. S. Wingreen, Science 280, 567 (1998).

${ }^{6}$ J. Li, W.-D. Schneider, R. Berndt, and B. Delley, Phys. Rev. Lett. 80, 2893 (1998).

7 T. W. Odom, J.-L. Huang, C. L. Cheung, and C. M. Lieber, Science 290, 1549 (2000).

${ }^{8}$ W. Hofstetter, J. Konig and H. Schoeller, condmat/0104497.

${ }^{9}$ Y. Meir and N. S. Wingreen Phys. Rev. Lett. 68, 2512 (1992).

${ }^{10}$ Y. Meir, N. S. Wingreen and P. A. Lee, Phys. Rev. Lett. 66, 3048 (1991).

11 The self-consistent equation is: $n_{\sigma}=\operatorname{Im} \int \frac{d \epsilon}{2 \pi} G_{\sigma}^{<}(\epsilon)$.

${ }^{12}$ Y. Meir, N. S. Wingreen and P. A. Lee, Phys. Rev. Lett. 70, 2601 (1993).

13 T.-K. Ng, Phys. Rev. Lett. 76, 487 (1996).

${ }^{14} \operatorname{LDOS}(\epsilon)=-\operatorname{Im} \sum_{\sigma} G_{\sigma}^{r}(\epsilon) / \pi$.

${ }^{15}$ R. Aguado and L. P. Kouwenhoven, Phys. Rev. Lett. 84, 1986 (2000).

FIG. 1. Two solid curves are the differential conductance $d I_{2} / d V_{2}$ versus $V_{2}$, with $\Gamma_{2}=\Gamma_{4}=0.001$ and $\gamma=0$. Two dotted curves are the LDOS versus energy $\epsilon$ for the two-probe objective system (obtained by setting $\Gamma_{2}=\Gamma_{4}=0$ ). The thick dashed curve is $d I_{2} / d V_{2}$ versus $V_{2}$ with $\Gamma_{2}=\Gamma_{4}=0.001$ and $\gamma=0.05$. Other parameters are: $\Gamma_{1}=\Gamma_{3}=1$, $V_{1}=-V_{3}=0.1$, and $T=0.005$. The thick curves and thin curves correspond to $\epsilon_{d \uparrow}(0)=\epsilon_{d \downarrow}(0)=-4.0$ and $\epsilon_{d \uparrow}(0)=-4.2, \epsilon_{d \downarrow}(0)=-3.8$, respectively. Notice that the dotted curves almost overlap perfectly with the solid curves so that they almost cannot be seen in the figure. The inset shows the two Kondo resonance peaks at zero magnetic field (by setting $\epsilon_{d \uparrow}(0)=\epsilon_{d \downarrow}(0)=-4.0$ ).
FIG. 2. $\quad d I_{2} / d V_{2}$ versus $V_{2}$ at different parameters $\gamma$. Other parameters are same as those of the thin solid line of Fig.1. The inset is a schematic diagram for the four-probe quantum dot device.

FIG. 3. $d I_{2} / d V_{2}$ versus $V_{2}$ for three different temperatures $T$, where the parameters are: $\Gamma_{1}=\Gamma_{3}=1$, $\Gamma_{2}=0.001, \gamma=0.05, \epsilon_{d \uparrow}(0)=-4.15, \epsilon_{d \downarrow}(0)=-3.85$, and $V_{1}=-V_{3}=0.15$. The inset is a schematic diagram for a proposed three-terminal quantum dot device. 


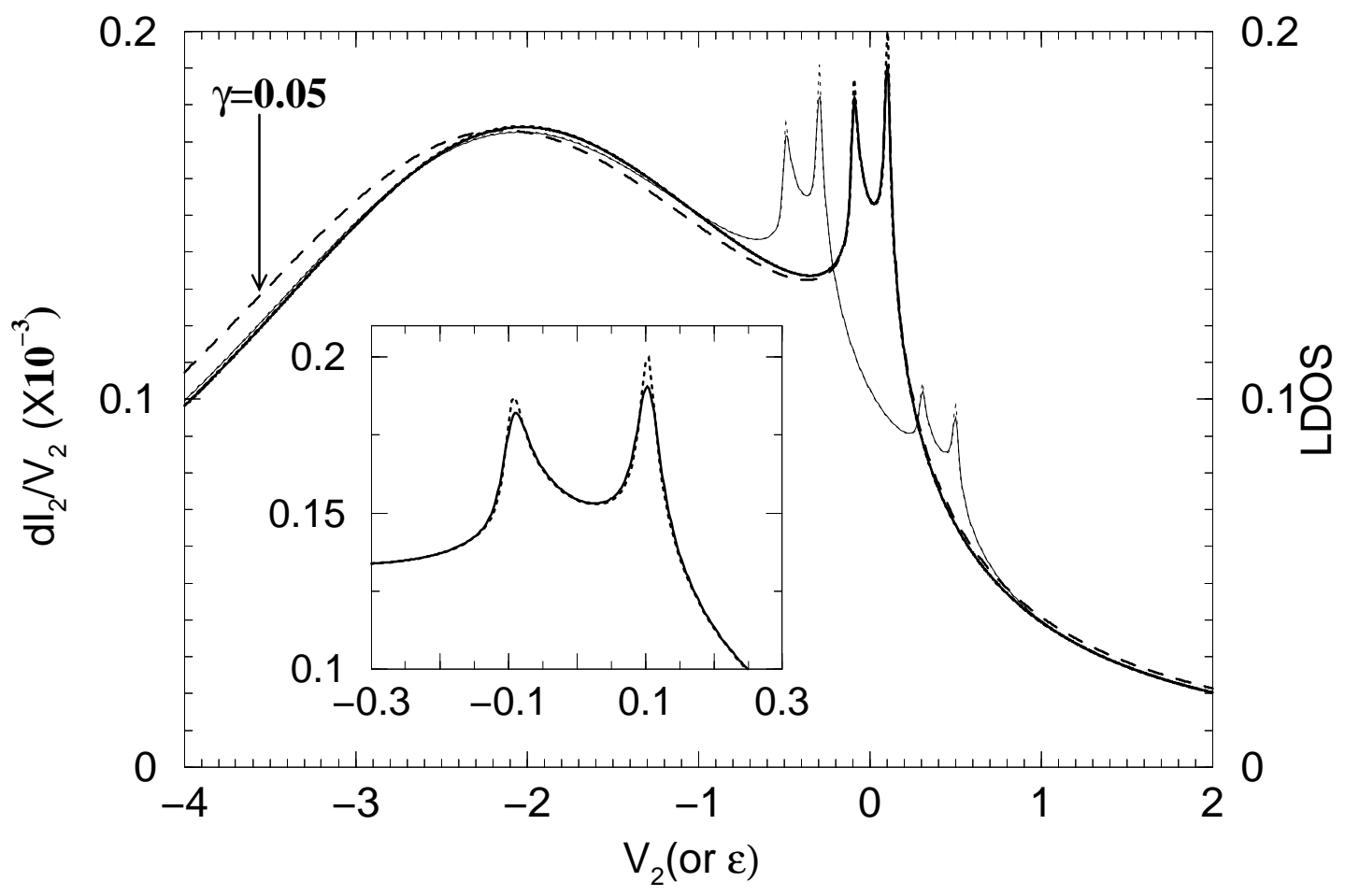

Fig.1 


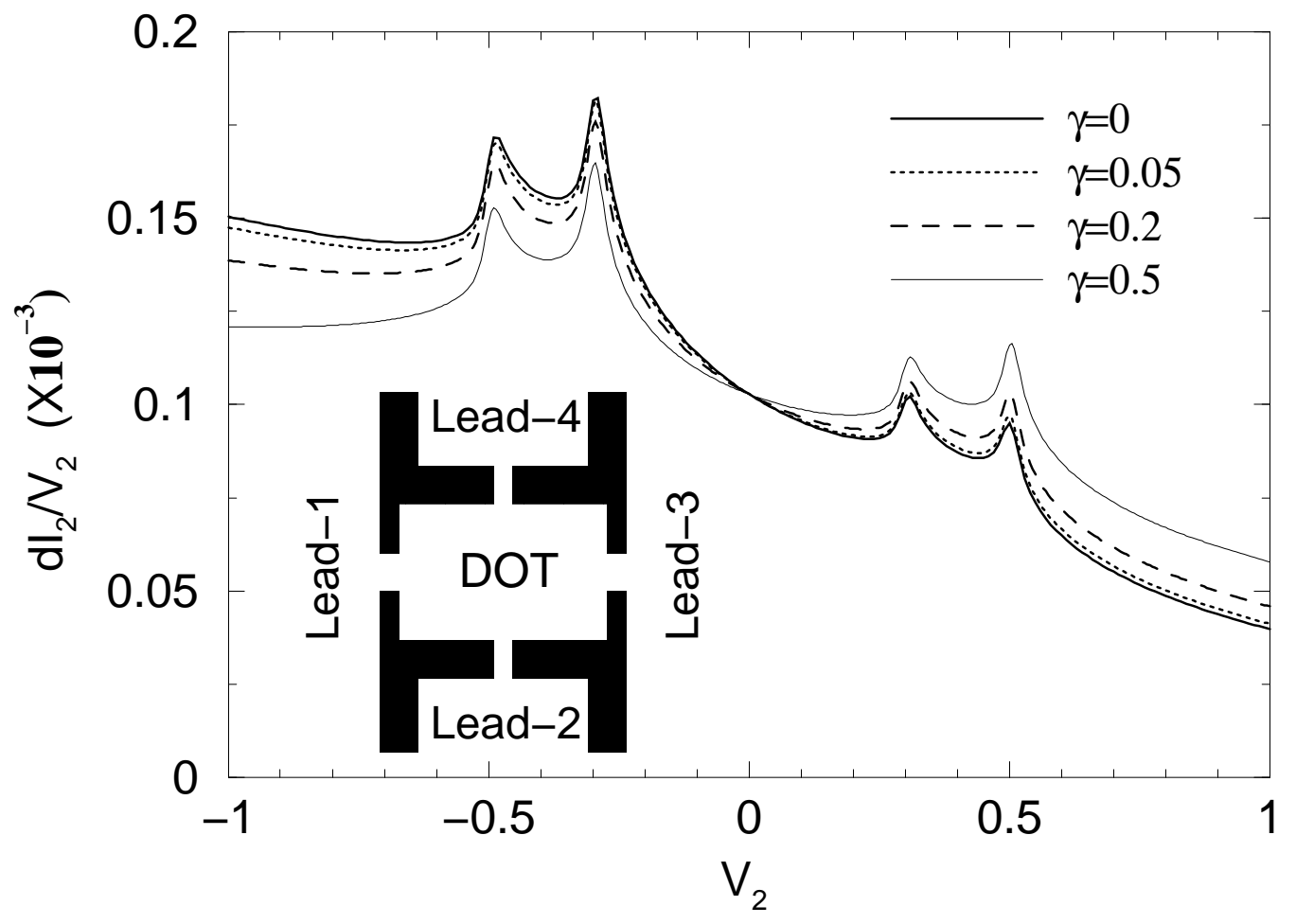

Fig.2 


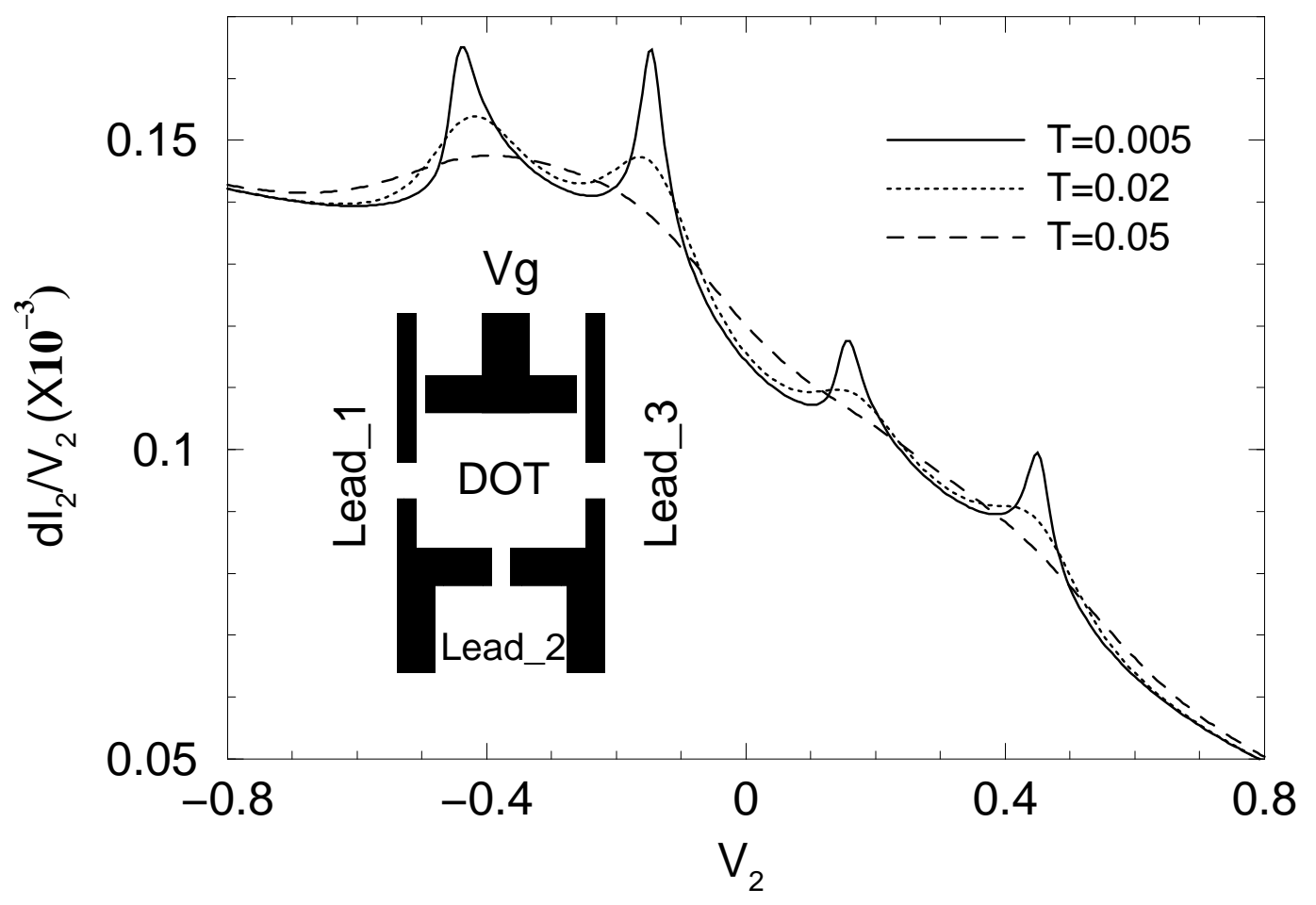

Fig.3 